\title{
Chemical profiling of polyphenols in Thunbergia alata and in silico virtual screening of their antiviral activities against COVID-19
}

\author{
Fatma S. Abd El -Aal ${ }^{*}$, Hala Sh. Mohammed ${ }^{1}$, Mona H. Ibrahim² and Lotfy D Ismaail ${ }^{3}$ \\ ${ }^{1}$ Department of Pharmacognosy, Faculty of Pharmacy (Girls), Al-Azhar University, Cairo, Egypt \\ ${ }^{2}$ Department of Pharmaceutical Chemistry, Faculty of Pharmacy (Girls), Al-Azhar University, Cairo, Egypt \\ ${ }^{3}$ Department of Pharmacognosy, Faculty of Pharmacy (Boys), Al-Azhar University, Cairo, Egypt
}

*Correspondence: E-mail: fs92020@gmail.com

Article history: Received 2021-04-18

\begin{abstract}
A comprehensive analysis of the polyphenols constituents found in Thunbergia alata leaves using (UPLC-Triple TOF-MS/MS) and natural polyphenols' antiviral properties have motivated us to perform in silico binding affinity study of flavonoids and coumarins identified in $T$. alata leaves extract against different proteins of SARS-COV-2; (Mpro) main protease (PDB ID: 6LU7) and 2'-O-methyltransferase (PDB ID: 6wkq) using a cocrystalized ligands N3 and Sinefungin respectively. 30 compounds were tentatively identified in $T$. alata leaves extract by UPLC-Triple TOF-MS/MS and characterized as 8-phenolic acids; 12-flavonoids; 3-coumarins and remain compounds unidentified. In silico virtual screening of both flavonoids and coumarins as the most common classes of polyphenols; flavonoids have the best potential to act as COVID-19 Mpro \& MTase inhibitors; especially Kaempferol-3-O-(6"' -p-coumaroyl)-glucoside; Comp. (17) which gave very best docking score $\{-9.2 \mathrm{kcal} / \mathrm{mol}\}$ (docking energy score of N3 $-7.6 \mathrm{kcal} / \mathrm{mol}$ ). It bind by nine hydrogen bonds (with TRY54, CYS145, GLN192, PHE140, LEU141, THR26, ARG188 and ASN142), and Pi-Sulfur interaction (with MET165), hydrophobic interactions via five Pi-Alkyl bond (with MET49, CYS145 and PRO168) and also scored energy binding -10.2 (docking energy score of Sinefungin $-7.8 \mathrm{kcal} / \mathrm{mol}$ ); formed H- bond with ASN6841, ASN6899, CYS6913, LYS6968, GLY6869, GLY6871, ASP6928, ASN6996 and TRY6930. Moreover, 17 binds with LYS6844, LYS6935, LEU6898 and CYS6913 via hydrophobic interactions. While coumarins in comparison with the current flavonoids had less energy binding score. However, further research is important to investigate their potential medicine value.
\end{abstract}

Key words: Thunbergia, COVID -19, Docking, Polyphenolics, UPLC-QTOF-ESI -MS/MS; Antioxidant

\section{INTRODUCTION}

Corona Virus Disease (COVID-19) is an RNA virus with glycoprotein spikes on its envelope soshow a crownlike appearance ${ }^{(1)}$. It is not the primarytime a coronavirus has caused an epidemic: in November 2019, a plague of coronaviruses (CoVs) with the intense acute respiratory syndrome (SARS)- CoV began within the Chinese province of Guangdong, and in September2012, the center East respiratory syndrome (MERS)-CoV emerged (2), (CoVs) are divided into four genera: (I) -coronavirus alpha $\mathrm{CoV}$ ), (II) coronavirus (beta $\mathrm{CoV}$ ), and (III) - coronavirus (delta $\mathrm{CoV}$ ), and (IV) -coronavirus (gamma $\mathrm{CoV}$ ), which are most likely found in bats and rodents ${ }^{(3)}$. A variety of active phytochemicals have been found to have genetically and functionally diverse therapeutic applications against the virus. Theantiviral mechanism of those agents is often explainedby their antioxidant activity, their scavenging capabilities, DNA inhibition, RNA synthesis, or inhibition of viral reproduction.
Numerous epidemiological and experimental studies have proven that antiviral actions work againsta large number of phytochemicals ${ }^{(4)}$. Polyphenols are one of the most abundant phytochemical constituents. They are known as phenolic acids, flavonoids, coumarins, lignans, stilbenes, and other comps basedon their chemical structure. Because of their molecular structures, they can be found in almost all plants and have a high antioxidant capacity. Polyphenols also possess a wide array of beneficial pharmacological activity. They are currently widely used to treat cancer, diabetes, cardiovascular disease, arthritis, neurodegenerative disorders, and a variety ofother diseases (5). T. alata is an herbaceous vine belonging to family Acanthaceae is one of natural plant cultivated in Egypt rich with polyphenols, Traditional medicine employs T. alata as fresh root extract is used as a tonic and aphrodisiac in India. Back and knee pains, eye inflammation, piles, and some ear disorders in cattle are all treated with this plant medicinally ${ }^{\left({ }^{(}\right)}$. T. alata leaves have been shownto be used 
to treat boils and other skin problems ${ }^{(7)}$.

This plant's leaf powder has anti-inflammatory effects. ${ }^{(8)}$, the whole plant extract have the hypoglycemic activity (9) and the anti-bacterial activity against Salmonella typhi and Pseudomonas aeruginosa was reported ${ }^{(10)}$ in spite of vast body of research concerning $T$. alata leaves, its antiviral activities is not clear. Thus, the study aims to use ultra-performance liquid chromatography coupled by mass spectrometry (UPLC$\mathrm{MS} / \mathrm{MS}$ ) to recognize polyphenolic constituents in $T$. alata leaves, as well as in silico screening of their antiviral activities against COVID-19.

\section{METHODS}

\subsection{Collection, identification and drying of Plant materials}

Fresh leaves of Thunbergia alata were collected from the Attia Shoala flower plantation in Kaliobeya, Egypt during May and June (2018) (flowering stage). The plant was kindly identified by Dr. Therese Labib, Botanical specialist, department of Flora and Taxonomy, El-Orman Garden, Giza, Egypt. A voucher specimen (Reg. No. T5) of the plant was deposited in the pharmacognosy department's herbarium at Al- Azhar University's Faculty of Pharmacy in Cairo, Egypt.

\section{Preparation of plant extract}

Maceration of air-dried powder (50g) in 70\% ethanol. $(3 \times 500 \mathrm{ml})$ at $(25 \pm 2){ }^{\circ} \mathrm{C}$., the extract was filtered, and the solvent was evaporated using a rotary evaporator (Buchi Co., Switzerland) at $50^{\circ} \mathrm{C}$. To obtain $100 \mathrm{mg}$ semidried extract for chemical profiling.

\subsection{UPLC-QTOF-MS/MS}

UPLC-QTOF-MS/MS was accustomed to investigate the secondary metabolites of $T$. alata leaves extract. The temperature of the LC system was set to room temperature. In $1 \mathrm{~mL}$ of mobile phase A $(5 \mathrm{mM}$ HCOONH4 buffer at pH8 in 1\% methanol), $50 \mathrm{mg}$ of extract was dissolved., vortexed for $2 \mathrm{~min}$ followed by ultra-sonication for $10 \mathrm{~min}$, centrifugation for $5 \mathrm{~min}$ at $10000 \mathrm{rpm}$ phase then $20 \mu \mathrm{l} \operatorname{stock}(50 / 1000 \mu \mathrm{l})$ re diluted with $1000 \mu \mathrm{l}$ reconstitution solvent. Finally, the injected concentration was $1 \mu \mathrm{g} / \mu \mathrm{l}$. The following multi-step linear gradient was applied mobile phase A ( $5 \mathrm{mM} \mathrm{HCOONH4}$ buffer $\mathrm{pH} 8$ in $1 \%$ methanol) with gradient increase from (10-90) \% of mobile phase B. (100\% acetonitrile) for 28 minutes with a rate of flow $0.3 \mathrm{ml} / \mathrm{min}$. Columns; pre column In-Line filter disks $0.5 \mu \mathrm{m} \quad \mathrm{x} \quad 3.0 \quad \mathrm{~mm}$ (Phenomenex), column X select HSS T3

$2.5 \mu \mathrm{m}, 2.1 \times 150 \mathrm{~mm}$ (waters) Column temperature 40

${ }^{\circ} \mathrm{C}$ were used for separation. Analyst software TF 1.7.1 (SCIEX) for LC-QTOF control, the injection volume in the UPLC system was $15 \mu \mathrm{L}$ for sample and mobile phase as the blank sample.

\section{RESULTS}

\subsection{UPLC-QTOF-ESI-MS/MS of polyphenolic compounds}

In the current study, the polyphenolic $2^{\text {ry }}$ metabolites in $T$. alata leaves ethanol extract were identified using UPLC-QTOF-ESI -MS/MS".The RT, full MS spectra, and $\mathrm{MS}^{\mathrm{n}}$ were used to determine the limit of detection for each peak of Compounds. By comparing reference compounds spectra and literature, fragmentation patterns in negative mode were revealed. 30 comps were identified tentatively in $T$. alata extract and characterized as phenolic acids; Caffic acid (1), 3,4-Dihydroxybenzoicacid (2),4-Hydroxy3-methoxy mandelate (3), Chlorogenic acid (4), Rosmarinic acid (7), Phlorizin (8), Astringene (16) \& pcoumaroylmalic acid (28). Flavonoids Daidzein-8- $C$ glucoside (6), Kaempferol- 3-O-Lrhamnoside (10), Baicalein-7-O-glucouronouide(11)

,Datiscin(12), Formononetin (13), Apigenin-7-Oglucoside (15), 4'-hydroxyisoflavone-7- $O$-glucoside (20) ,Kaempferol-7- neohesperidoside (26), 3'Methoxy4',5,7trihydroxy-flavone (27), Myricetin (29) and Isorhamnetin-3-O rutinoside (30). Coumarins;Scopoletin(14) Delphnetin (19) and Esculin (23). Fatty acid; Gamma Linolenic acid (25). [ Figure 1 \& Table 1].

\subsection{Molecular Docking}

All docked molecules were establish to anchored closely in the active binding site of main protease (Mpro) and $\left(2^{\prime}\right)$ O-RNA methyltransferase (MTase) enzymes of SARS-CoV-2 with range of binding energies (-5.4 to -9.2) $\mathrm{kcal} / \mathrm{ml}$ and (-6.1 to-10.2,) $\mathrm{kcal} / \mathrm{mol}$, respectively (Supplementary data; Table S, Figure. S1 and S2). Flavonoids have the best potential to act as COVID-19 Mpro \& MTase inhibitors; especially Kaempferol-3-O(6"' -p-coumaroyl)-glucoside (fig. 2, 3).

\section{DISCUSSION}

\subsection{Annotation of polyphenolics using UPLC- QTOF- MS/MS}

\section{Flavonoids}

Comp. 6 was detected at Rt $2.90 \mathrm{~min}$, with deprotonated ion $[\mathrm{M}-\mathrm{H}]$ - at $\mathrm{m} / \mathrm{z} 415.16$, it could be identified as Daidzein-8-C- glucoside (Puerarin) (11). Comp. 10 detected at Rt $5.47 \mathrm{~min}$, showed a deprotonated ion [M-H]- at $\mathrm{m} / \mathrm{z} 431.23$ and MSn ions were observed at $\mathrm{m} / \mathrm{z}$ 385.18, 223.13 and 153.09 , it was tentatively identified as Kaempferol-3-O-L- rhamnoside ${ }^{(12)}$ Comp. 11 detected at Rt $5.75 \mathrm{~min}$, showed a molecular weight $414 \mathrm{~g} / \mathrm{mole}$. Ions were observed at $\mathrm{m} / \mathrm{z} 415.17,299.6$, $269.04 \& 179.06$,

it was tentatively identified as Baicalein-7-Oglucouronide (Baicalin) ${ }^{(13)}$. Comp. 12; was detected at $\mathrm{R}_{\mathrm{t}}$ $6.26 \mathrm{~min}$, showed a deprotonated ion [M-H] ${ }^{-}$at $\mathrm{m} / z 593.16$ and $\mathrm{MS}^{\mathrm{n}}$ ions were observed at $\mathrm{m} / z$ 549.1, 539.3, 447.10 and 285.03 which identified 431.09 and MSn ions were 
observed at $\mathrm{m} / \mathrm{z} 269.04266 .03$ which identified tentatively as apigenin-7- $O$-glucoside ${ }^{(16)}$, Comp. 20 was detected at $\mathrm{R}_{\mathrm{t}} 10.66 \mathrm{~min}$, showed a deprotonated ion $[\mathrm{M}-\mathrm{H}]-$ at $\mathrm{m} / \mathrm{z}$ 299.21 characteristic for molecular weight $230 \mathrm{~g} / \mathrm{mol}$ and MSn ions were observed at $\mathrm{m} / \mathrm{z} 266.04,258.04229 .04$, 210.08, 203.02, 146.11and 128.06 which identified tentatively as3, 5, 7-trihydroxy-4'- methoxyflavanone ${ }^{(19)}$. Comp. 22 was detected at $\mathrm{R}_{\mathrm{t}} 13.47 \mathrm{~min}$, showed a deprotonated ion $[\mathrm{M}-\mathrm{H}]^{-}$at $\mathrm{m} / \mathrm{z} 283.06$ and [M-2H-CH3] at $\mathrm{m} / \mathrm{z} 266.03$ which tentatively identified as acacetin ${ }^{(19)}$. Comp. 24was detected at $\mathrm{R}_{\mathrm{t}} 15.59 \mathrm{~min}$, showed a deprotonated ion $[\mathrm{M}-\mathrm{H}]^{-}{ }^{-}$at $m / z 413.8$ which characteristic formolecular weight $414 \mathrm{~g} / \mathrm{mole}$. So comp. 24 tentatively identified $^{(20)}$. Comp. 29 was detected at $\mathrm{R}_{\mathrm{t}}$

$23.20 \mathrm{~min}$, showed a deprotonated ion $[\mathrm{M}-\mathrm{H}]^{-}$at $\mathrm{m} / \mathrm{z}$ 317.12, and $\mathrm{MS}^{\mathrm{n}}$ ions were observed at $\mathrm{m} / \mathrm{z} 248.9$, 180.9 and 116.6which tentatively identified as myricetin $^{(21)}$.

\section{Coumarins}

Comp. 14 was detected at $\mathrm{R}_{\mathrm{t}} 7.44 \mathrm{~min}$, showed a deprotonated ion [M-H] $]^{-}$at $m / z$ 191.02and $\mathrm{MS}^{\mathrm{n}}$ ions were observed at $\mathrm{m} / \mathrm{z} \quad 175.90$ and 131.01which tentatively identified as Scopoletin $^{(22) \&(14)}$. showed a deprotonated ion [M-H] $]^{-}$at $m / z$ 177.05and $\mathrm{MS}^{\mathrm{n}}$ ions were observed at $\mathrm{m} / \mathrm{z}$ $162.03145 .025 .03131 .01 \quad 121.02 \quad 118.04117 .03$ which tentatively identified as Daphnetin (7,8Dihydroxycoumarin) (23), Comp. 23 was detected at $\mathrm{R}_{\mathrm{t}}$ $14.71 \mathrm{~min}$, showed a deprotonated ion $[\mathrm{M}-\mathrm{H}]^{-}$at $\mathrm{m} / z$ 339.08 and $\mathrm{MS}^{\mathrm{n}}$ ionswere observed at $\mathrm{m} / z 293.21,177.0$ and 149.0 which tentatively identified as Esculin (6,7-Dihydroxycoumarin 6-glucoside) ${ }^{(24) \text {. }}$

\subsection{Molecular Docking}

The Protein Data Bank (https://www.rcsb.org/) was used to obtain the crystal structures main protease of COVID-19 (Mpro) (PDB ID: 6LU7) ${ }^{(25)}$. And 2'-

Omethyltransferase (PDB ID: 6wkq) ${ }^{(26)}$. Autodock Vina 4.2 was used to perform the docking study,which requires both the ligand and the receptor to be in pdbqt extension. Prior to the docking, M.G.L werethe tools used for preparing the two enzymes, as wellas the co-crystalized and two lead compounds, in theproper format ${ }^{(27)}$. By redocking the co-crystalized ligands into their complexed enzymes, the measured RMSD between the docked and co- crystalized ligands was less than $0.67 \AA$, suggestingthat the docking method was valid. The dockingresults were visually inspected using the Discovery Studio 4.5 visualizer $^{(28)}$.

\subsubsection{Docking into main protease of COVID-19}

Various research groups have focused into the (Mpro) of SARS-CoV-2, also known as 3CLpro (chymotrypsin-like protease, as a possible drug targetto treat COVID-19. It is essential for replication of RNA viruses because it aids in the translation and maturation of viral RNA, providing it an engaging target for drugs against coronavirus ${ }^{(29)}$. 3CLpro composed of 9 alphahelices and $13 \beta$-strands, with 3 distinct domains (Domain I, II, III) with an extended loop. The catalytic dyad of the SARS-CoV-2 virus consists of conserved amino acid residues CYS145 and HIS41, and the determination of binding site of substrate is created by splitting Domain I and II ${ }^{(30)}$. All docked molecules were established to anchored closely in the active binding site of COVID-19 3CLpro with range of binding energies (-5.4 to -9.2) $\mathrm{kcal} / \mathrm{mol}$ (Supplementary data). The flavonoids act as a protease inhibitor especially Comp. (17) which showed the highest docking score $-9.2 \mathrm{kcal} / \mathrm{mol}$ It formed nine $\mathrm{H}$ bonds (with TRY54, CYS145, GLN192, PHE140, LEU141, THR26, ARG188 and ASN142), and Pi Sulfur interaction (with MET165), in addition to hydrophobic interactions via five Pi Alkyl bond (with MET49, CYS145 and PRO168) Fig. 2. On the other hand, N3 formed seven hydrogen bonds (with CYS 145, GLN 189, THR26 and HIS 163), Pi-Anion with GLU166, Pi-Sigma with HIS 41 and two alkyl interactions with MET49 and MET165fig. 2. Additionally, there were another eight flavonoid comp.s with lower binding energy than thenative ligand in binding affinity ranged from $-7.8 \mathrm{kcal} / \mathrm{mol}$ (comp.s 18 and 6), -7.9 $\mathrm{kcal} / \mathrm{mol}$ (comp. 24),-8.1 kcal/mol ( comp. 15 ),-8.2 $\mathrm{kcal} / \mathrm{mol}$ (comp. 11),- 8.3kcal/mol ( comp. 10), -8.7 $\mathrm{kcal} / \mathrm{mol}$ (comp. 12)and - $8.9 \mathrm{kcal} / \mathrm{mol}$ (comp. 29 ). Whereas the coumarin comps. 14,19 and 23 observed that has less energy binding equal $-5.4,-5.9$ and -7.2 respectively to conclude that coumarins less protease inhibitor than flavonoids (Figure. 2 and supplementary data).

\subsubsection{Docking into SARS-CoV- 2- (2') O RNA methyltransferase (MTase);}

A potential target for antiviral therapy is (MTase)which is needed for formation of RNA cap, that required for stability of viral RNA ${ }^{(31)}$. It belongsto a large class of SAM-dependent methyltransferases, this MTase feature is binding to the nsp-16 protein, that needs a cofactor nsp-10, to function properly. ASP6928, LYS6839, LYS6968, and GLU7001 are highly conserved residues in protein nsp-16 that form the catalytic canonical motif (KD- K-E) conserved among class I MTases ${ }^{(32)}$ 


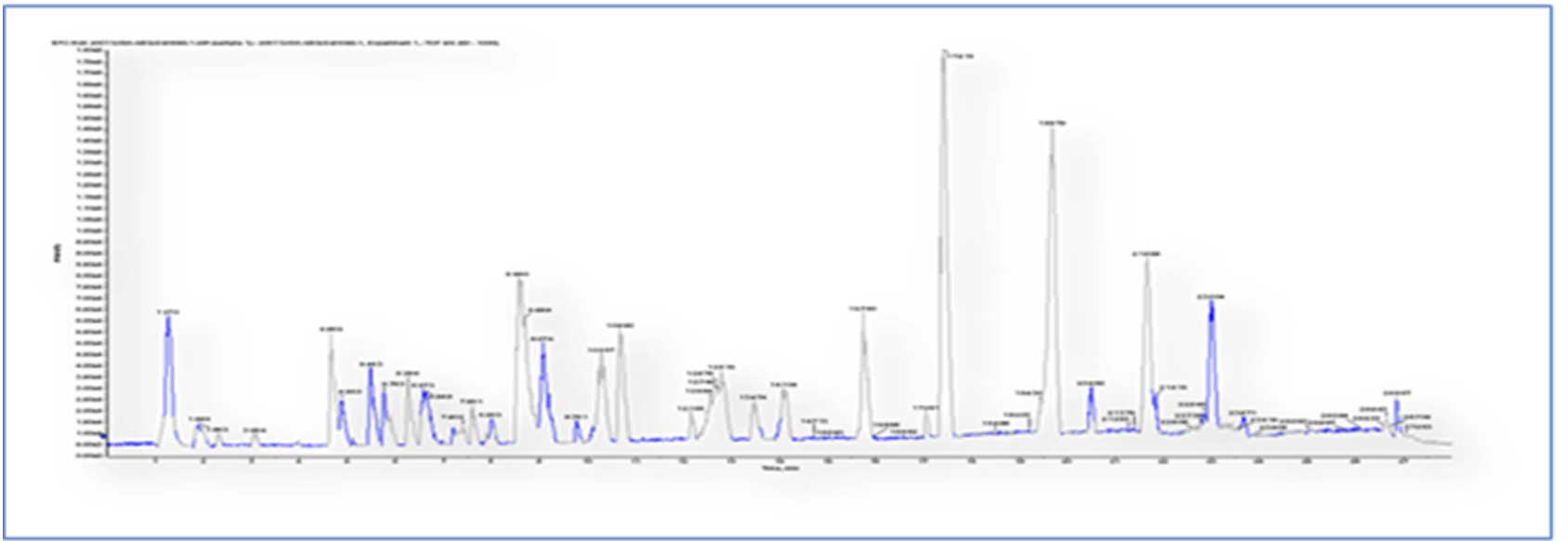

Figure (1): Negative mode UPLC-QTOF-ESI-MS/MS chromatogram of leaves. Peak numbers agree with those in Table (1).

Table (1): Tentatively identified polyphenol comp.s by negative mode UPLC-QTOF-MS/MS in T. alata leaves ethanolextract.

\begin{tabular}{|c|c|c|c|c|}
\hline No & RT & {$[\mathbf{M}-\mathbf{H}]^{-}$} & Major product ions (m/z) & Tentatively identified compounds \\
\hline 1. & 1.23 & 179.03 & Not fragmented & Caffeic acid \\
\hline 2. & 1.24 & 153.02 & $109.03,98.79$ & 3,4-Dihydroxybenzoic acid \\
\hline 3. & 1.31 & 197.03 & $151.06,137.02,122.03$ & 4-Hydroxy-3-methoxymandelate \\
\hline 4. & 1.90 & 353.09 & $288.03,207.05,199.80,179.03$ & Chlorogenic acid \\
\hline 5. & 1.97 & 346.10 & $189.06,181.08$ & Unidentified \\
\hline 6. & 2.90 & 415.16 & Not fragmented & Daidzein-8-C-glucoside \\
\hline 7. & 4.65 & 359.07 & $197.6,179.03,161.02,133.02$ & Rosmarinic acid \\
\hline 8. & 4.87 & 435.10 & Not fragmented & Phlorizin \\
\hline 9. & 5.47 & 345.15 & Not fragmented & Unidentified \\
\hline 10. & 5.47 & 431.23 & $285.18,223.13,153.09$ & Kaempferol-3-O-L-rhamnoside \\
\hline 11. & 5.75 & 445.17 & $415.17,299.60,269.04,179.06$ & Baicalein-7-O-glucouronide \\
\hline 12. & 6.26 & 593.16 & $549.10,539.30,447.10,285.03$ & Datiscin \\
\hline 13. & 6.66 & 267.08 & $252.07,193.10,180.03, \quad 155.07,149.05133 .02$ & Formononetin \\
\hline 14. & 7.44 & 191.02 & $175.90,131.01$ & Scopoletin \\
\hline 15. & 7.59 & 431.09 & $269.04, \quad 266.03$ & Apigenin-7-O-glucoside \\
\hline 16. & 8.60 & 405.16 & 225.11 & Astringene \\
\hline 17. & 9.07 & 593.12 & 309.09 & Kaempferol-3-O-(6' ' '-p-coumaroyl)-glucoside \\
\hline 18. & 10.28 & 269.05 & $\begin{array}{l}240.03, \quad 177.06,162.03,159.03145 .02,121 . \\
117.02\end{array}$ & Apigenin \\
\hline 19. & 10.28 & 177.05 & $162.03,145.02,131.01,121.02,118.04,117$ & Daphnetin \\
\hline 20. & 10.66 & 299.21 & $\begin{array}{l}266.04,258.04,229.04,210.08,203.02,146.1 \\
128.06\end{array}$ & 3, 5, 7-trihydroxy-4'-methoxyflavanone \\
\hline 21. & 12.16 & 467.22 & $399.25,179.06,143.03$ & Unidentified \\
\hline 22. & 13.47 & 283.06 & 266.03 & Acacetin \\
\hline 23. & 14.71 & 339.08 & $293.21,177.0,149.0$ & Esculin \\
\hline 24. & 15.59 & 413.80 & Not fragmented & 4'-hydroxyisoflavone-7-O-glucoside \\
\hline 25. & 17.06 & 277.21 & $210.01,151.02$ & gamma Linolenic acid \\
\hline 26. & 19.86 & 593.14 & 473.11 & Kaempferol-7-neohesperidoside \\
\hline 27. & 20.49 & 315.21 & $300.04,273.08,269.24,256.10 \quad 178.95,148$ & 3'-Methoxy-4',5,7-trihydroxy flavone \\
\hline 28. & 21.60 & 279.23 & 261.22 & p-coumaroyl-malic acid \\
\hline 29. & 23.2 & 317.12 & $248.90,180.89,116.6$ & Myricetin \\
\hline 30. & 26.63 & 623.2 & 616.5 & Isorhamnetin-3-O-rutinoside \\
\hline
\end{tabular}



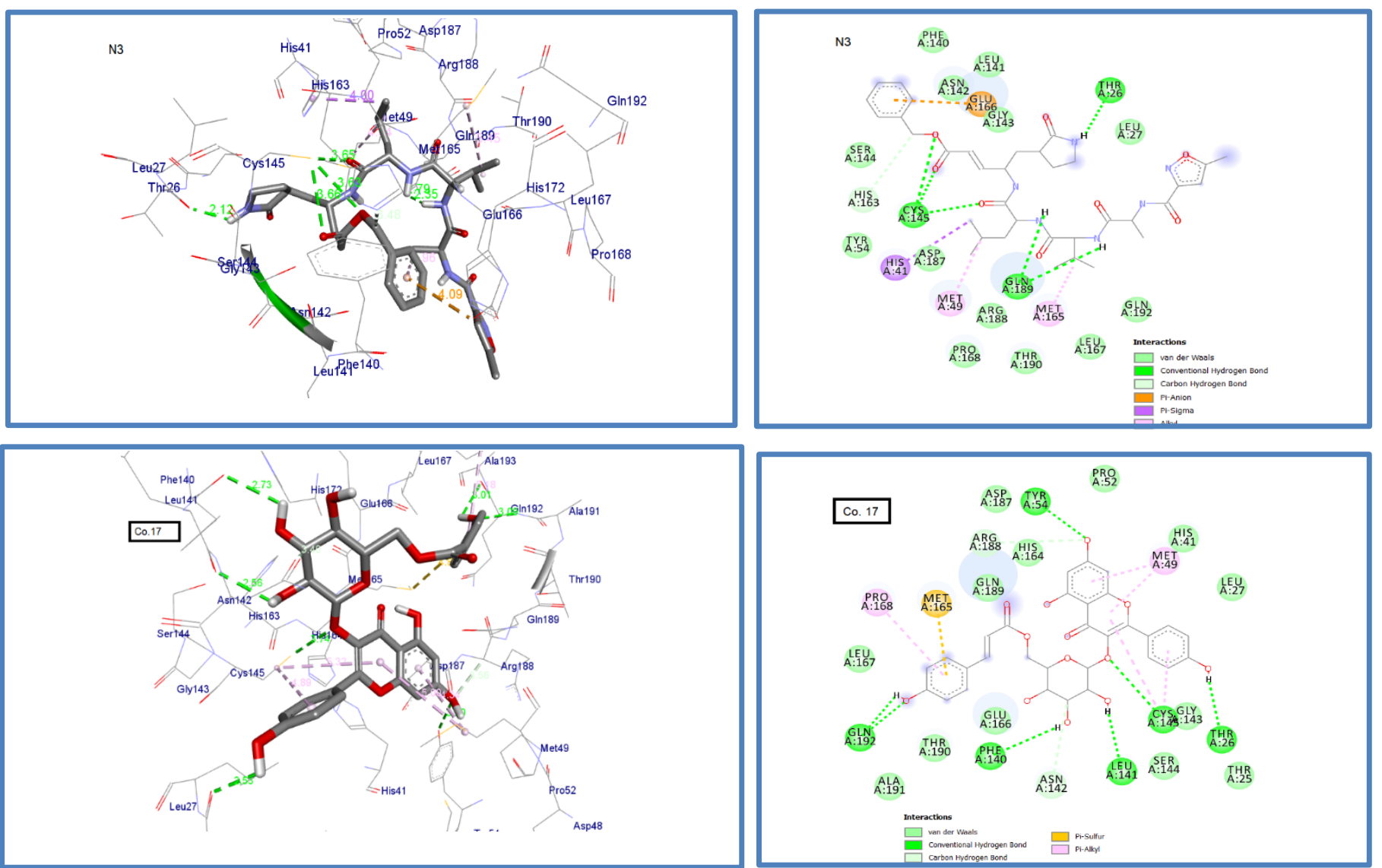

Figure 2: 2D and 3D visualization of molecular interaction of 6LU7 protease with N3 and comp. 17
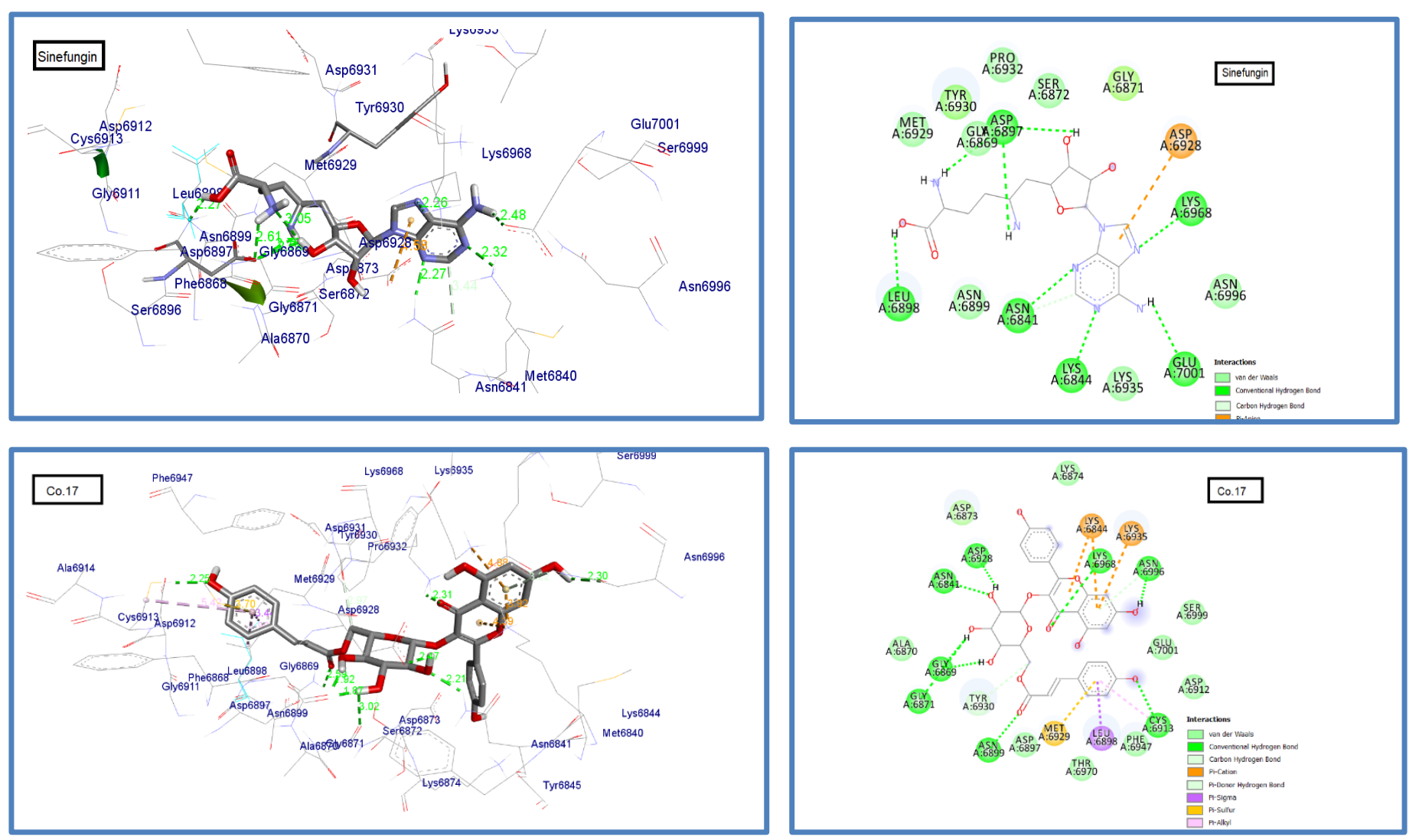

Figure 3: 2D and 3D visualization of molecular interaction SARS-CoV-2 2' $O$-RNA MTase with Sinefungin and comp. 17. 
Residues LEU6898, ASP6912, CYS6913, MET6928, and PHE6947 stabilize the adenosine moiety of SAM in the nsp-10-nsp-16 complex crystal structure bound to the pan- MTase inhibitor sinefungin (PDB: $6 \mathrm{WKQ})$ at 2.0-A in the active site. SAM's sugar moiety interacts with GLY6871 andASP6897 residues, as well as two water molecules that interact with ASN6899, and the interaction of methionine moiety with ASN6841, GLY6871ASP6928, and TYR6845, Sinefungin is in the same manner as SAM does ${ }^{(30)}$. The energy of binding is obtained from docking $6 \mathrm{wkq}$ with Sinefungin, compounds $14,19,23,10,17,12,29,15,22,11,18,20,24,13,6$, and 29 were $-7.8,-6.1,-6.7,-7.4,-7.7,-10.2,-8.8,-8.9,-9.5$, $7.6,-8.7,-8.8,-7.3,-8.8,-8.4,-9.3$ and $-9.3 \mathrm{kcal} / \mathrm{mol}$, respectively (Supplementary data). Docking analysis (Table 1) showed that all compounds fit in the correct binding site. Whereas comp. 17 showed also thehighest binding score, formed $\mathrm{H}$ bond with ASN6841, ASN6899, CYS6913, LYS6968, GLY6869, GLY6871, ASP6928, ASN6996 and TRY6930. Moreover 17 binds withLYS6844, LYS6935, LEU6898 and CYS6913via hydrophobic interactions. (Supplementary data, Figure. 3).

\section{CONCLUSION}

Flavonoids, are a wide group of polyphenolic compounds with a benzo- $\gamma$-pyrone structure, are used to prevent and cure diseases, and have significant biological activity. Many flavonoids have been shown to be effective antiviral agents in clinical trials. Kaempferol-3-O-(6"'-p-coumaroyl) - glucoside; in the current study showed thehighest energy binding score to achieve the best naturalantiviral remedy, so it should be interesting to separate it using chromatographic techniques and detected by authentic sample, 1D and 2D NMR spectroscopy for further in-vivo and in-vitro research as a natural antiviral remedy.

\section{List of abbreviations \\ COVID-19}

SARS-CoV- 2-

3CLpro

Mpro

2' O-RNA MTase

T. alata

UPLC-QTOF

$\mathrm{MS} / \mathrm{MS}$

$\mathrm{m} / \mathrm{z}$
Coronavirus disease

Severe acute respiratory

syndrome coronavirus 2

Chymotrypsin-like protease

Main protease

(2') O RNA methyltransferase

Thunbergia alata

Ultra-performance liquid

chromatography quadrupole

time of flight mass

spectrometry

Major product ions

\section{REFERENCE}

1. Perlman S, Netland J, Coronaviruses post-SARS: Update on replication and pathogenesis. Nat Rev Microbiol. 2009; 7(6): 439-450.

2. Prof Roujian Lu Ms, Xiang Zhao M, Juan Li P, Peihua Niu P Bo, Yang Ms Honglong, Wu Ms et al. Genomic characterisation and epidemiology of 2019 novel coronavirus: implications for virus origins and receptor binding. NCBI.2020; 395(10224): 565-74

3. Yin Y, Wunderink RG MERS, SARS and other coronaviruses as causes of pneumonia. Resp. 2018; Feb; 23(2):130-7.

4. Muthu B, Janarthanan AK. Antiviral activity of herbal plants and its phytocomp.s(an overview). Mukt Shabd Jour.2020; 2347-3150.

5. Sinha D, Pharmacological Importance of Polyphenols a Review. Inter. Res. Jour. Of Pharmacy. 2019. 10: 13-23.

6. Ram M, Rao K, Alam A, Singh KS, Ram M, Rao K. Preliminary phytochemical analysis of different extracts of Ruellia patula Luffa aegyptiaca and Thunbergia alata. Chem. and Pharm. Res. 2015; 7(10):315-20.

7. Rojas Sandoval J, Acevedo Rodiguez P. Thunbergia alata (black eyed Susan) [Internet]. I S C. 2018. Available from: https://www.cabi.org/isc/datasheet/53646\#tocont ributor.

8. Cho YC, Kim YR, Kim BR, Bach TT, Cho S. Thunbergia alata inhibits inflammatory responses through the inactivation of ERK and STAT3 in macrophages. Vol. 38, Inter. J. of Mol. Med. 2016: 1596-604.

9. Charles A, Ramani VA. Chemosymantics of genus Tunbergia IJSRME. 2016; 2455 - 5630:511.

10. KH S, S C. Ethnopharmacological and phytochemical review on Thunbergia Retz. (Montin.) Species. Med Aromat Plants 2015; 04:217

11. Jeevan K. P, Kenneth J, Marion K, Landon W, Michelle S-J, Connie W Profiling and Quantification of Isoflavonoids in Kudzu Dietary Supplements by HPLCand ElSI-TMS. 2 J. Agric. Food Chem. 2003; 51(15): 4213-18

12. Rivière C, Hong VNT, Pieters L, Dejaegher B, Heyden Y Vander, Van MC, et al. Polyphenols isolated from antiradical extracts of Mallotus metcalfianus. Phytochemistry. 2009; 70(1):8694.

13. Yuan Y, Hou W, Tang M, Luo H, Chen LJ, Guan $\mathrm{YH}$, et al. Separation of flavonoids from the 
leaves of Oroxylum indicum by HSCCC.Chromatographia. 2008;68:885-92.

14. Tine Y, Renucci F, Costa J, Wélé A, Paolini J. A method for LC-MS/MS profiling of coumarins in zanthoxylum zanthoxyloides (Lam.) B. Zepernich and timler extracts and essential oils. Molecules. 2017; 22; 22(1):174.

15. Zhao X, Zhang S, Liu D, Yang M, Wei J. Analysis of Flavonoids in Dalbergia odorifera by UltraPerformance Liquid Chromatography with Tandem Mass Spectrometry. Molecules. 2020; 25(2):389.

16. Ana Plazonić, Bucar F, Maleŝ Ẑ, Mornar A, Nigović B, Kujundẑić N. Identification and Quantification of flavonoids and phenolic acids in burr parsley (caucalis platycarpos L.), using high-performance liquid chromatography with diode array detection and electrospray ionization mass spectrometry. Molecules. 2009;14 :246690 .

17. Seon-H L, Heon-W, Min-KL, Young J K, Gelila A Y-SC et al. Phenolic profiling and quantitative determination of common sage ( Salvia plebeia R. Euro Food Res. and Tech. 2018;244(9):1637-46

18. María de la Luz Cádiz-Gurrea, Salvador F-A, Jorge J, Antonio S-C. Comprehensive characterization by UHPLC-ESI-Q-TOF-MS from an Eryngium bourgatii extract and their antioxidant and anti-inflammatory activities. 2013; 09. (38): 197-204.

19. Sawada Y, Nakabayashi R, Yamada Y, Suzuki M, Sato M, Sakata A, et al. RIKEN tandem mass spectral database (ReSpect) for phytochemicals: A plant-specific MS/MS-based data resource and database. 2012; 82:38-45.

20. Anna S, Barbara S, Joanna B, Dorota M MJ, MS. LC_MS profiling of flavonoid glycoconjugates isolated from hairy roots, suspension root cell cultures and seedling roots of Medicago truncatula . Metabolomics. 2011; 7: 604-13.

21. Venkatalakshmi $\mathrm{P}$, Vadivel V, Brindha $\mathrm{P}$. Identification of flavonoids in different parts of terminalia catappa L. Using LC-ESI-MS/MS and investigation of their anticancer effect in EAC cell line model. Pharm.Sci.and Res. 2016; 8: 176-83.

22. Siwinska1 J, Kadzinskil L, Banasiuk1 R, Gwizdek-Wisniewskal A, Alexandre O, Banecki1 B, et al. Identification of QTL affecting scopolin and scopoletin biosynthesis in Arabidopsis thaliana. BMC Plant Bio .2014; 14:280.

23 Yefchak V L. Mass Spectral Fragmentation Studies of Coumarin-Type Comp.s Using GC High-Resolution MS. Analyt. Chem. 2011; 5:
27-36.

24 Ying-L, YingS C,Liu X, HuangX Z Neng L M , XuSui M N ,Sheng W. Simultaneous determination of esculin and its metabolite esculetin in rat plasma by LC-ESI-MS_MS and its application in pharmacokinetic study -. J Chromatogr B Analyt Technol Biomed Life Sci .2012 Oct 15; 907:27-33.

25. Jin Z, Du X, Xu Y, Deng Y, Liu M, Zhao Y, et al. Structure of Mpro from SARS-CoV-2 and discovery of its inhibitors. Nature. 2020; 582(7811):289-93.

26. Rosas-Lemus M, Minasov G, Shuvalova L1, Inniss NL, Kiryukhina O BJ, Satchell KJF. Highresolution structures of the SARS-CoV-2 2'methyltransferase reveal strategies for structurebased inhibitor design. Science Signaling .2020; 13: 651 .

27. Oleg T, Arthur J. O. AutoDock Vina: Improving the Speed and Accuracy of Docking with a New Scoring Function, Efficient Optimization, and Multithreading. Journal of comp. chem. 2010; 31: 455-61.

28. Mahmoud A. H, Tamer M. I, Sara T. R, Amal Alharbi ROE\& WME. In silico identification of novel SARS-COV-2 ${ }^{-}$'-O-methyltransferase (nsp16) inhibitors structure-based virtual screening,.molecular dynamics simulation and MM-PBSA approaches. 2021; 36(1): 727-36.

29. Mothay D, Ramesh K V. Binding site analysis of potential protease inhibitors of COVID-19 using AutoDock. VirusDisease. 2020; 31(2):194-9.

30. Sang P, Tian SH, Meng ZH, Yang LQ. Anti-HIV drug repurposing against SARS-CoV-2. RSC Adv. 2020; 10(27):15775-83.

31. Krafcikova P., Silhan J., Nencka EB R.Discovery of Neutralizing Antibody (NAb) and Peptide Targeting SARS-CoV - Creative Biolabs. 2020.

32. Rosas-Lemus M, Minasov G, Shuvalova L, Inniss N, Kiryukhina O, Brunzelle J, et al. Structure of SARS-CoV-2 2'-O-methyltransferase heterodimer with RNA Cap analog and sulfates bound reveals new strategies for structure-based inhibitor design. Nature. 20201. 